\title{
Physico-chemical nurturing of the 'Cell'- A tool for Rejuvenation
}

\section{Samuel JK Abraham*}

The microenvironment within the body where stem cells reside and are nurtured (the stem cell niche) in various organs including that of Hematopoietic stem cells (HSCs) in the bone marrow (BM) has always been a challenge when it comes to understanding their intricacies. But, as we travel back in time to that of unicellular organisms in evolution, the nature per se was the niche for those unicellular organisms, where they were in direct contact with naturally occurring substances like rocks, soil, water etc. In the process of evolution, as unicellular organisms evolved to become multicellular, the term niche became a separate entity, internally specific to each organ within that organism (1). Cells in the unicellular organisms were directly exposed to physical and chemical influences of the nature and after developing into multicellular organisms with specific organs, the influence of physico-chemical factors became indirect. Nevertheless, physical forces and chemical moieties do play a role either directly or indirectly in every organism and their internal niche.

In vitro cell or tissue culture in the earliest recorded history was done using simple chemical solutions, mainly balanced salt solutions (2) which were later modified to include biological components as well $(3,4)$. In recent times as more evidences have started gathering, chemically defined synthetic media is used for the cell culture (5) and the influence of physical forces on the cell culture has also started becoming more evident (6). Our experience till date proves that chemically synthesised polymers and nanomaterial based scaffolds could allow an in vitro expansion of the cells such as corneal limbal stem cells (7), corneal endothelial precursors (8) and chondrocytes (9) in the laboratory, which not only avoids potential threat of biological material based contamination but also ensures reproducibility as well as simplicity.

The process of rejuvenation using in vitro expanded cells has been our major area of interest, having been equipped with contamination free, easily reproducible cell culture methodologies of different types of cells. While in vitro expanded autologous fibroblast replenishment (10) has been able to bring back a youthful facial skin, cellular senescence under various physical and chemical environments is an important area for future research. In vitro expansion of immune cells such as Natural Killer (NK) cells and T cells without animal derived feeder layers is a proven procedure for treating cancer (11) and studies have been reported wherein such immune cells could bring down the viral load (12). The hematopoietic stem cells with known age related damages when could be cultured in vitro and be cryopreserved without the damages that are inflicted during the process of aging in vivo, may open new avenues for total rejuvenation of the human body.

\section{REFERENCES:}

http://www.metaniche.org/

Miller DJ. J Physiol. 2004; 555:585-7

Harrison R. Anat Rec. 1907 1:116-128.

Harrison R. J Exp Zool. 1910; 9:787-846.

Ham RG. Proc Natl Acad Sci U S A. 1965; 53:288-93.

Engler AJ et al. Cell. 2006;126:677-89.

Sitalakshmi G et al. Tissue Eng Part A. 2009;15:407-15.

Rao $\mathrm{S}$ et al. Indian Journal of Ophthalmology (In print)

Arumugam S et al. J.Orthopaedics 2011; 8(3)e5.

http://www.miawmi.com/

Takada M et al. Breast Cancer. 2011;18:64-7.

Zhang T et al. Antiviral Res. 2007;73:132-9.

\footnotetext{
* Dr. Samuel JK Abraham, Nichi-In Centre for Regenerative Medicine (NCRM), India \& Yamanashi University Hospital, Japan
} 